\title{
COMUNICACIÓN
}

\section{Trichomona vaginalis en la mujer Aymara residente en zona urbana}

\author{
MARIA TERESA MONTALVO*, CELIA BÓRQUEZ*, ISMELDA LOBATO*, \\ TERESA REYES* e HILDA VILLANUEVA*.
}

\section{Trichomona vaginalis INFECTION IN AYMARA WOMAN RESIDENT IN URBAN AREAS}

There is an ethnic Aymara group with well defined sociocultural characteristics in the highlands of the $1^{o}$ region of Chile. They have tendency towards the endogamy, which they have sustained through time and in the last years they have had moved towards the urban sector. Due to emigration it is important to know the prevalence the Trichomona vaginalis on this ethnic group, resident in the urban zone of Arica. The study was performed in 95 Aymara women in fertile age which had emigrated to the urban zone. In every woman a gynaecological exam was performed and a sample of vaginal secretions was taken for the determination of $\boldsymbol{T}$. vaginalis. The results from these studies showed that the prevalence the $\boldsymbol{T}$. vaginalis was of $10.5 \%$. In relation to the infection grade by age, the most common porcentage showed between 20 to 29 years old. The $11.1 \%$ of women with T. vaginalis begin its sexual activity between 16 and 25 years of age. Sexual promiscuous is the $40 \%$ and from them the $15.8 \%$ was in infected. Beetween the woman which remain social cultured attached to their natural habitat only $4.9 \%$ was infected. We can conclude that the Aymara woman migrating and establishing in the urban sector, without keeping bonds with their origin place, received the impact the urban medium provoking the increase of the $\boldsymbol{T}$. vaginalis.

Key words: Trichomona vaginalis infection, Aymaras, survey.

\section{INTRODUCCIÓN}

Actualmente a nivel mundial existe un incremento importante y sostenido de las Infecciones de Transmisión Sexual (ITS), que afecta a todos los grupos sociales y étnicos de nuestra sociedad, especialmente entre los $15 \mathrm{y}$ 30 años ${ }^{1}$.

La tricomoniosis, es una de las enfermedades de transmisión sexual común y persistente del aparato genito urinario, causada por Trichomona vaginalis, con una incidencia anual estimada en casi doscientos millones de mujeres en todo el mundo, con mayor incidencia entre las mujeres de 16 a 35 años y alta prevalencia entre mujeres con múltiples parejas sexuales ${ }^{2-5}$.

En la Primera Región de Chile, existe el grupo étnico aymara, de especial importancia por sus características socioculturales y geográficas, cuyo origen se remonta aproximadamente al siglo $\mathrm{V}$ antes de Cristo, caracterizado por un sistema social cerrado, formado por agricultores, pescadores y pastores, que mantienen una dinámica de desarrollo y crecimiento típicamente

* Departamento de Biología y Salud, Facultad de Ciencias, Universidad de Tarapacá. Casilla D-7. Arica-Chile. Email: maritemontal@hotmail.com 
nómade y que en busca de mejores condiciones de vida, ha emigrado a la ciudad de Arica $^{6-8}$.

En nuestro país se han realizado numerosos estudios para conocer aspectos clínicos, epidemiológicos y de diagnóstico de la tricomoniosis urogenital ${ }^{9-14}$. Un estudio realizado en mujeres aymara, habitantes del altiplano y precordillera chilena de las provincias de Arica y Parinacota, mostró una baja prevalencia de $T$. vaginalis ${ }^{15}$. Este hallazgo motivó el estudio de la prevalencia de este parásito en el mismo grupo étnico, pero que residiera en la zona urbana de Arica.

\section{MATERIAL Y MÉTODOS}

La muestra estuvo constituida por 95 mujeres aymara, entre 15 y 49 años, residentes en la ciudad de Arica, que concurrían a control ginecológico en el Servicio Municipal de Salud de Arica y estaban aparentemente sanas.

Se consideró aymara a aquellas mujeres cuyos apellidos y el sus padres tenían ese origen, además, de sus rasgos físicos, criterios que han sido utilizados por este y otros grupos de investigadores en investigaciones sobre esta etnia, como también por otros investigadores. Para esto se tomó como base documentos que contienen antecedentes étnicos, como también todos los apellidos de los ancestros de los individuos de ese origen ${ }^{16,17}$.

A cada mujer seleccionada se le solicito autorización, debidamente informada, para participar en el estudio. Se tomó una muestra de secreción vaginal del orificio cervical externo y fondo del saco vaginal, según técnica recomendada por el Instituto de Salud Pública (ISP) ${ }^{18}$, para el estudio de observación directa y cultivo en medio Diamond. Además se confeccionó una ficha epidemiológica, con antecedentes tales como, edad actual, edad de inicio de la actividad sexual y grado de promiscuidad.

\section{RESULTADOS}

El resultado de infección por $T$. vaginalis en las 95 mujeres en zona urbana se muestra en la Tabla 1. En la Tabla 2 se muestra la frecuencia de infección en la mujer aymara urbana en relación a la edad. La frecuencia de infección en relación a la edad de inicio de la actividad sexual se observa en la Tabla 3 y la Tabla 4 muestra su frecuencia en relación a la promiscuidad sexual de la mujer aymara urbana.

Tabla 1. Frecuencia de Trichomona vaginalis en la mujer aymara residente en zona urbana. Arica, 2001

\begin{tabular}{lcc}
\hline Infección & $\mathbf{n}$ & $\boldsymbol{\%}$ \\
\hline Infectadas & 10 & 10,5 \\
No Infectadas & 85 & 89,5 \\
Total & 95 & 100 \\
\hline
\end{tabular}

Tabla 2. Frecuencia de Trichomona vaginalis en la mujer aymara en zona urbana, según edad. Arica, 2001

\begin{tabular}{lccc}
\hline $\begin{array}{l}\text { Grupos } \\
\text { Etáreos (años) }\end{array}$ & $\begin{array}{c}\mathbf{N}^{\mathbf{0}} \\
\text { Estudiado }\end{array}$ & $\begin{array}{c}\mathbf{N}^{\mathbf{0}} \\
\text { Infectadas }\end{array}$ & $\begin{array}{c}\% \\
\text { infectadas }\end{array}$ \\
\hline $15-19$ & 3 & 0 & 0 \\
$20-24$ & 19 & 3 & 30 \\
$25-29$ & 34 & 4 & 40 \\
$30-34$ & 17 & 1 & 10 \\
$35-39$ & 12 & 1 & 10 \\
$40-44$ & 5 & 1 & 10 \\
$45-49$ & 5 & 0 & 0 \\
Total & 95 & 10 & 100 \\
\hline
\end{tabular}

Tabla 3. Frecuencia de Trichomona vaginalis en la mujer aymara residente en zona urbana, en relación a la edad de inicio de la actividad sexual. Arica, 2001

\begin{tabular}{lccc}
\hline $\begin{array}{l}\text { Edad inicio de } \\
\text { la actividad } \\
\text { sexual (años) }\end{array}$ & $\begin{array}{c}\mathbf{N}^{\mathbf{0}} \\
\text { Estudiadas }\end{array}$ & $\begin{array}{c}\mathbf{N}^{\mathbf{2}} \\
\text { Infectadas }\end{array}$ & $\begin{array}{c}\% \\
\text { infectadas }\end{array}$ \\
\hline$<15$ & 5 & 0 & 0 \\
$16-20$ & 72 & 8 & 11,1 \\
$21-25$ & 12 & 2 & 1,7 \\
$26-30$ & 1 & 0 & 0 \\
\hline
\end{tabular}

Tabla 4. Frecuencia de Trichomona vaginalis en relación a la promiscuidad sexual de la mujer aymara en zona urbana. Arica, 2001

\begin{tabular}{lccr}
\hline \multicolumn{2}{c}{ Promiscuidad } & $\mathbf{N}^{\mathbf{o}}$ & \multicolumn{2}{c}{$\mathbf{N}^{\mathbf{0}}$} & $\begin{array}{c}\text { \% } \\
\text { Sexual }\end{array}$ & Estudiadas & Infectadas & infectadas \\
\hline $\mathrm{Si}$ & 38 & 6 & 15,8 \\
No & 57 & 4 & 7,0 \\
\hline
\end{tabular}


Trichomona vaginalis en la mujer aymara residente en zona urbana - M. T. Montalvo et al.

Tabla 5. Frecuencia de Trichomona vaginalis en relación a la mantención de vínculos con su lugar de origen en la mujer de origen aymara. Arica, 2001

\begin{tabular}{lccr}
\hline $\begin{array}{l}\text { Mantiene } \\
\text { vínculos }\end{array}$ & $\begin{array}{c}\mathbf{N}^{\mathbf{0}} \\
\text { estudiadas }\end{array}$ & $\begin{array}{c}\mathbf{N}^{\mathbf{2}} \\
\text { infectadas }\end{array}$ & $\begin{array}{c}\% \\
\text { infectadas }\end{array}$ \\
\hline Sí & 41 & 2 & 4,9 \\
No & 54 & 8 & 14,8 \\
\hline
\end{tabular}

\section{DISCUSIÓN}

Como puede observarse en la Tabla 1, de un total de 95 mujeres aymara estudiadas residentes en la ciudad de Arica, se encontraron 10 casos de $T$. vaginalis $(10,5 \%)$, lo que representa un porcentaje estadísticamente más alto a lo reportado en la mujer aymara en su habitat natural $(3,7 \%)$, en el Altiplano y Precordillera ${ }^{15}$, lo que indica que la mujer aymara tiende a adaptarse a su nueva condición de vida, este valor es inferior al promedio nacional, que es aproximadamente de $20 \%{ }^{2}$ y de alrededor de un $12 \%$ reportado en otras poblaciones ${ }^{19,20}$.

En relación al grado de infección según la edad, los mayores porcentajes se encuentran en mujeres entre 20 a 29 años, (Tabla 2). Es importante señalar que la mayor incidencia de agentes productores de ITS se presenta en el periodo de mayor actividad sexual del individuo $^{1,9,19}$. Los resultados encontrados son similares a los obtenidos en la mujer aymara residente en el altiplano y precordillera ${ }^{15}$.

En cuanto a la edad de inicio de la actividad sexual, la Tabla 3 , muestra que el $11,1 \%$ de la frecuencia de $T$. vaginalis corresponde al grupo que inicio su actividad sexual entre los 16 y 25 años. Al comparar estos resultados con lo obtenido en la mujer aymara residente en su habitat natural se encontró que estas inician más tempranamente su actividad sexual (15 hasta 20 años) con un porcentaje de infección menor $(5,3 \%)^{15}$, lo anterior indicaría que la mujer aymara al migrar a la ciudad tarda en adaptarse a las costumbres urbanas.

La Tabla 4 indica el grado de promiscuidad sexual en la mujer aymara así como la influencia que tiene ésta en relación a la presencia de $T$. vaginalis. Los valores encontrados con respecto a esta variable indican, por un lado, que la promiscuidad sexual en la mujer aymara es alta (40\%), con respecto a lo reportado en la mujer en el altiplano y precordillera que es sólo un $16 \%$ y siendo este un factor predisponente importante en las ITS, explicaría la presencia que 6 de los 10 casos encontrados $(15,8 \%)$, se hallaran en esta condición.

Además, es importante destacar que la mujer aymara que emigra al sector urbano y se radica en él no manteniendo vínculos con su lugar de origen recibe el impacto del medio urbano incidiendo en el aumento de la tricomoniosis, ya que esta es un enfermedad de exclusiva transmisión sexual, lo cual hace suponer un cambio en su conducta sexual, lo anterior se reafirma al observar que la mujer aymara que mantiene vínculos con su lugar de origen $(43,2 \%)$ presenta sólo un $4,9 \%$ de infección, valor que contrastra con el $14,8 \%$ que sí mantienen vínculos (Tabla 5).

\section{RESUMEN}

En el altiplano de la I Región de Chile existe un grupo étnico aymara con características socioculturales bien definidas, con gran tendencia a la endogamia la que han mantenido sostenidamente en el tiempo y que en los últimos años ha emigrado al sector urbano. Este hecho hizo importante conocer la prevalencia de Trichomona vaginalis en este grupo étnico, que reside en la zona urbana de Arica. El estudio se efectuó en 95 mujeres aymaras en edad fértil que han emigrado al sector urbano. A cada una de ellas se le realizó examen ginecológico y toma de muestra de secreción vaginal para estudio de $T$. vaginalis.

Los resultados obtenidos muestran un $10,5 \%$ de prevalencia de T. vaginalis. En relación al grado de infección según edad los mayores porcentajes se encuentran en mujeres entre 20 y 29 años. El $11,1 \%$ de la mujeres con $T$. vaginalis corresponde al grupo que inicio su actividad sexual entre los 16 y 25 años. La promiscuidad sexual es de un $40 \%$ y de ellas el 15,8\% estaban infectadas. Las mujeres que mantienen vínculos con su lugar de origen están infectadas en un 4,9\%.

Se puede concluir que la mujer aymara que emigra al sector urbano y se radica en él, no manteniendo vínculos con su lugar de origen, recibe el impacto del medio urbano incidiendo en el aumento de T. vaginalis, ya que esta al ser una infección exclusivamente de transmisión sexual, hace suponer un cambio en su conducta sexual. 


\section{REFERENCIAS}

1.- BERNAL J. Las Enfermedades de Transmisión Sexual. Rev Chil ETS 1986; 1: 4-5.

2.- REYES V. Tricomoniasis. En: Parasitología Clínica. Eds. A. Atías pp 367 - 373 Editorial Mediterráneo. Santiago. 1992.

3.- BENENSON A. Tricomoniasis. En: Manual para el control de las enfermedades transmisibles. pp 461462 Organización Panamericana de la Salud. 1997.

4.- VILLALOBOS D. Enfermedades de Transmisión Sexual. Proyecto ETS en Chile. Ministerio de Salud. 1995.

5.- GILBERT D, VEREECKEN A, BOSMANS E et al. Definition of a type of abnormal vaginal flora that is in distinct from bacterial vaginosis aerobic vaginitis. Int J Obst Gynaecol. England 2002; 109: 34-43.

6.- IRARRÁZABAL D. Boletín del Instituto de Estudios Aymaras. 1988.

7.- SANTORO C, UlloA L. Culturas en Arica. Departamento de Extensión Cultural del Ministerio de Educación. 1990.

8.- GUNDERMANN H. Ganadería Aymara. Ecología y Forrages. Rev Chungara 1986; 12: 99-124.

9.- REYES H, LYNCH B, NOEMÍ I et al. Estudio sobre Trichomona vaginalis, hongos y bacterias en muestras de flujo vaginal. Rev Méd Chile 1974; 102: 279-82.

10.- APT W, MILOS A, ASPEE I et al. Estudio Cínico y de laboratorio del flujo vaginal en 191 mujeres. Rev Méd Chile 1975; 103: 754-8.

11.- SAGUA H, FUENTES A, RABY M. Diagnóstico de la trichomoniasis: Evaluación de técnicas de recolección y transporte de las muestras. Rev Méd Chile 1983; 11:
909-12.

12.- SAGUA H, CHIANG B, VARELA H et al. Tricomonosis en adolescentes embarazadas de Antofagasta, Chile. Parasitol al Día 1999; 23: 9-14.

13.- MERCADO R, BASALDUA J, MADARIAGA A. Frecuencia de infección por Trichomona vaginalis en mujeres aparentemente sanas del área occidente de Santiago. Bol Hosp S J de Dios 1985; 32: 88-9.

14.- FRANJOLA R, AÑAZCO R, PUENTE R et al. Trichomona vaginalis en adolescentes embarazadas y recién nacidos. Rev Méd Chile 1989; 117: 142-5.

15.- LOBATO L, MONTALVO MT, BÓRQUEZ C et al. ETS en mujeres aymaras del altiplano de la Primera Región. Chile. ETS Rev Chil Enf Tran Sex 1991; 6: 80-6.

16.- HIDALGO L J. Revisita de los Altos de Arica en 1750. Publicación de la U. del Norte. Chile. Departamento de Antropología. 1978.

17.- CHIPANA C, Origen y significado de nuestros apellidos. Instituto Regional de Promoción Aymara. Arica. 1990.

18.- GARCÍA J, CASTILLO L, BUSTOS R et al. Diagnóstico de laboratorio de las infecciones del tracto genital femenino y masculino. En: Procedimientos técnicos de laboratorio clínico. Instituto de Salud Pública 1994. pp 41-6.

19.- PRADO R, OGETA A, OJEDA B et al. Frecuencia de infección por Trichomona vaginalis en mujeres chilenas aparentemente sanas. Bol Chil Parasitol 1976; 31: 43-4.

20.- ROJAS L, SOLANO R, SARIEGO I. Frecuencia de trichomoniasis vaginal en mujeres supuestamente sanas. Rev Cub Hig Epidemiol 2000; 37: 66-70. 